\title{
Functional Bowel Disorders in Patients with Brugada Syndrome and Drug-Induced Type 1 Brugada Pattern
}

\author{
Anil Sarica ${ }^{1}$, Serhat Bor ${ }^{1}$, Mehmet Orman ${ }^{1}$, Hector Barajas-Martinez ${ }^{2}$, Jimmy Juang ${ }^{3}$, \\ Charles Antzelevitch ${ }^{2}$, and Can Hasdemir ${ }^{1}$ \\ ${ }^{1}$ Ege University Faculty of Medicine \\ ${ }^{2}$ Lankenau Institute for Medical Research \\ ${ }^{3}$ National Taiwan University
}

December 30, 2020

\begin{abstract}
Introduction: Irritable bowel syndrome (IBS) is one of the most widely recognized functional bowel disorders (FBDs) with a genetic component. SCN5A gene and SCN1B loci have been identified in population-based IBS cohorts and proposed to have a mechanistic role in the pathophysiology of IBS. These same genes have been associated with Brugada syndrome (BrS). The present study examines the hypothesis that these two inherited syndromes are linked. Methods and Results: Prevalence of FBDs over a 12 months period were compared between probands with BrS/drug-induced type 1 Brugada pattern (DI-Type1 $\mathrm{BrP})(\mathrm{n}=148)$ and a control group $(\mathrm{n}=124)$ matched for age, female sex, presence of arrhythmia and co-morbid conditions. SCN5A/SCN1B genes were screened in 88 patients. Prevalence of IBS was $25 \%$ in patients with BrS/DI-Type1 BrP and $8.1 \%$ in the control group $(\mathrm{p}=2.34 \times 10-4)$. On stepwise logistic regression analysis, presence of current and/or history of migraine (OR of $2.75 ; 95 \%$ CI: 1.08 to $6.98 ; \mathrm{p}=0.033$ ) was a predictor of underlying BrS/DI-Type1 BrP among patients with FBDs. We identified 8 putative SCN5A/SCN1B variants in 7 (12.3\%) patients with BrS/DI-Type1 BrP and 1 (3.2\%) patient in control group. Five out of $8(62.5 \%)$ patients with SCN5A/SCN1B variants had FBDs. Conclusion: IBS is a common co-morbidity in patients with BrS/DI-Type1 BrP. Presence of current and/or history of migraine is a predictor of underlying BrS/DI-Type1 BrP among patients with FBDs. Frequent co-existence of IBS and BrS/DI-Type1 BrP necessitates cautious use of certain drugs among the therapeutic options for IBS that are known to exacerbate the Brugada phenotype.
\end{abstract}

\section{Hosted file}

Manuscript.pdf available at https://authorea.com/users/386247/articles/501739-functionalbowel-disorders-in-patients-with-brugada-syndrome-and-drug-induced-type-1-brugadapattern 